\title{
The Impact of Organisational Characteristics of Staff and Facility on Infectious Disease Outbreaks in Care Homes: a Systematic Review
}

\author{
Ann Liljas ( $\square$ ann.liljas@ki.se ) \\ Karolinska Institutet \\ Lenke Morath \\ Karolinska Institutet \\ Bo Burström \\ Karolinska Institutet \\ Pär Schön \\ Karolinska Institutet \\ Janne Agerholm \\ Karolinska Institutet
}

\section{Research Article}

Keywords: Nursing homes, elderly, care workers

Posted Date: September 13th, 2021

DOI: https://doi.org/10.21203/rs.3.rs-861018/v1

License: (c) (1) This work is licensed under a Creative Commons Attribution 4.0 International License. Read Full License

Version of Record: A version of this preprint was published at BMC Health Services Research on March 15th, 2022. See the published version at https://doi.org/10.1186/s12913-022-07481-w. 


\section{Abstract}

Background: Infectious disease outbreaks are common in care homes, often with substantial impact on the rates of infection and mortality of the residents, who primarily are older people vulnerable to infections. There is growing evidence that organisational characteristics of staff and facility might play a role in infection outbreaks however such evidence have not previously been systematically reviewed. Therefore, this systematic review aims to examine the impact of facility and staff characteristics on the risk of infectious disease outbreaks in care homes.

Methods: Five databases were searched. Studies considered for inclusion were of any design reporting on an outbreak of any infectious disease in one or more care homes providing care for primarily older people with original data on: facility size, facility location (urban/rural), facility design, use of temporary hired staff, staff compartmentalizing, residence of staff, and/or nursing aides hours per resident. Retrieved studies were screened, assessed for quality, and analysed employing a narrative synthesis.

Results: Sixteen studies (8 cohort studies, 6 cross-sectional studies, 2 case-control) were included from the search which generated 10,424 unique records. COVID-19 was the most commonly reported cause of outbreak $(n=11)$. The other studies focused on influenza, respiratory and gastrointestinal outbreaks. Most studies reported on the impact of facility size $(n=11)$ followed by facility design $(n=4)$, use of temporary hired staff $(n=3)$, facility location ( $n=2)$, staff compartmentalizing $(n=2)$, nurse aides hours $(n=2)$ and residence of staff $(n=1)$. Findings suggest that urban location and larger facility size may be associated with greater risks of an infectious outbreak. Additionally, the risk of a larger outbreak seems lower in larger facilities. Whilst staff compartmentalizing may be associated with lower risk of an outbreak, staff residing in highly infected areas may be associated with greater risk of outbreak. The influence of facility design, use of temporary staff, and nurse aides hours remains unclear.

Conclusions: This systematic review suggests that larger facilities have greater risks of infectious outbreaks, yet the risk of a larger outbreak seems lower in larger facilities. Due to lack of robust findings the impact of facility and staff characteristics on infectious outbreaks remain largely unknown.

PROSPERO: CRD42020213585

\section{Introduction}

Infectious disease outbreaks are common in shared living spaces such as care homes [1]. Infection outbreaks in care homes often have a substantial impact on the rates of infection and mortality of the residents who primarily are frail older people with chronic physical, mental and/or cognitive conditions and thus more vulnerable to infections [2,3]. The most common types of infectious disease outbreaks in care homes are respiratory infections such as influenza viruses, and gastrointestinal infectious (often caused by noroviruses) [4]. Additionally, in the last 1.5 years numerous care homes worldwide have been severely impacted by COVID-19 (SARS-CoV-2), an acute respiratory syndrome with worse outcomes for older adults with multimorbidity compared to younger adults [5]. In several countries such as the United States, England, France, Spain, and Sweden, care home residents have been reported to account for 30-50\% of all COVID-19-related deaths [6-8].

The prerequisites for infection control vary between care homes (also known as nursing homes or residential long-term care facilities) and depend on a range of factors including organisational factors such as facility characteristics (i.e. the physical building) [9] and staff characteristics [10]. In the last few decades there has been a shift towards building care homes with a homelike atmosphere [11]. The homelike environment has however been reported an obstacle in infection prevention and control [12]. Unlike hospitals, there are no transaction windows into the residents' apartments and there is rarely a designated space where employees can remove personal protective equipment or get changed after having had close personal contact with an infected resident. Besides providing a homelike atmosphere, care homes are often designed to bring people together for social activities and meals, routines that might have to change during an infection outbreak, with consequences such as loneliness and anxiety among the residents [13]. The design of care homes also affects the possibility of isolating residents or conducting cohort care to prevent transmission of infection during an outbreak. Handling residents with dementia or mental illness who have a strong compulsion to walk, known as 'wanderers', becomes particularly challenging when practising isolation and social distancing [14]. Earlier systematic reviews focusing on facility characteristics have shown that residents in care homes with a higher proportion of private rooms have reported better quality of life [15], and that care homes with fewer beds scored higher on service quality [16]. Findings on links between smaller facility size and residents' quality of life were however inconsistent [15]. The latter systematic review further reported higher quality of life among the residents in care homes in rural areas [15]. However, no systematic review has investigated the impact of these and other facility characteristics such as the facility design on infectious outbreaks. Further, in a brief summary of evidence on how pandemic spreads can be contained in care homes, the authors concluded that movement of staff between care homes should be limited, and that temporary staff are a key potential source of infection [17]. Similarly, a systematic review has reported higher risks of infection in healthcare settings with fewer registered nurses and more nursing aide staff, and with a higher proportion of temporary staff [18]. In the wake of the COVID-19 pandemic, the possible effects of care home staff, particularly nurse aides, working in multiple facilities on infection control has been debated [19]. Staff characteristics in relation to outbreaks of infectious diseases in care homes have however not previously been systematically reviewed. The impact of staff and facility characteristics are less studied aspects in healthcare research [20], yet considered potential modifiable factors for infection control in care homes $[9,10]$. This includes community-acquired infectious diseases such as COVID-19 where the spread in the local community might result in infected care workers being the probable source of outbreaks in care homes [21]. Identifying factors of particular importance for infection control in the home care setting is essential to mitigate the spread of infectious diseases. Therefore, we have conducted a systematic review that aims to examine the impact of organisational features such as staff characteristics and facility characteristics on the risk of infectious disease outbreaks in care homes for older adults.

\section{Methods}




\section{Search strategy}

Five electronic databases were searched for this review: MEDLINE (Ovid), EMBASE, Sociological Abstract (ProQuest), Web of Science Core Collection and CINAHL (Ebsco). The systematic search was performed in April 2021. Key search terms were developed in collaboration with librarians and included long term care, infection, and disease outbreaks, and are presented in Appendix 1. The research protocol has been registered with PROSPERO (identification number CRD42020213585).

\section{Eligibility criteria}

In this review, the outcome was an infectious disease outbreak in a care home, referring to one or more residents being infected. A single case of an infectious disease can be considered an outbreak if the disease is rare or has serious public health implications [22]. Table 1 presents the PICO (Population, Intervention, Comparison, Outcome) inclusion and exclusion criteria for eligible studies. Studies included were of quantitative, qualitative and mixed-method design reporting on an infectious disease outbreak in one or more care homes providing care for primarily older adults ( $\geq 65$ years) with original data on one or more of the exposure features: facility size (typically measured as number of beds), facility location (urban/rural), the design or room layout of the facility, temporary hired staff, staff compartmentalizing (staff working only with isolated/non-isolated residents), residence of staff (home address of staff), and nursing aides hours per resident (number of hours certified nurse aides are available to care for residents and undertake administrative work). The seven exposure features assessed were chosen based on facility and staff characteristics identified by the authors prior to the database search, and not previously systematically reviewed. The search was not restricted to certain years or languages. Reviews and commentaries with no original data were excluded.

Table 1

PICO

\begin{tabular}{|ll|}
\hline $\begin{array}{l}\text { Population } \\
\text { (Setting) }\end{array}$ & $\begin{array}{l}\text { Care homes for primarily older adults (aged } \geq 65 \text { years) defined as a facility with } 24 \text { hours surveillance and access to some level of } \\
\text { medical care within the facility. }\end{array}$ \\
\hline $\begin{array}{l}\text { Intervention } \\
\text { (Exposure) }\end{array}$ & $\begin{array}{l}\text { Facility size, facility location, facility design, use of temporary hired employment, staff compartmentalizing, residence of staff, and } \\
\text { nurse aides hours per resident. }\end{array}$ \\
\hline Comparison & No restriction \\
\hline Outcome & $\begin{array}{l}\text { An infectious disease outbreak (at least } 1 \text { resident infected in the care home facility). Studies that included disease outbreaks of non- } \\
\text { infectious or non-communicable diseases were excluded. }\end{array}$ \\
\hline
\end{tabular}

\section{Study selection, data extraction and quality assessment}

References retrieved were downloaded to Endnote X9 Reference Management and Rayyan for Systematic Reviews and independently screened for eligibility by two researchers (LM and AL). Any disagreements were resolved through discussion between the reviewers and a third researcher (JA). Researchers LM and AL extracted the data together and organised the studies according to study outcome using a standardised data extraction form. Studies providing findings from both cross-sectional analysis and findings from multiple data collection time points, data from the latter option were sought. Together the two researchers then assessed the studies for quality using the Critical Appraisal Skills Programme (CASP) (https://casp-uk.net/casp-tools-checklists/). The appraisal tool is used to analytically evaluate whether the results are valid, biases have been minimised and confounding factors have been considered, with the answer options yes/no/can't tell. Studies that were given one or more 'no' or 'can't tell' answers in the quality assessment were read again and discussed with the third researcher. If any 'no' or 'can't tell' answers remained, the study was considered not to be of high quality and subsequently excluded. Results on each exposure feature in each individual study were summarised and presented in tables (Tables 3 and 4 ). A narrative synthesis allowed for the findings of the heterogenous studies included in the review to be compiled.

\section{Results}

\section{Results of the systematic search}

A PRISMA flow chart of the screening process is presented in Fig. 1. The search yielded 15,786 records and after removal of deduplications 10,424 records remained, which were screened for eligibility. Two hundred and three studies were read in full text of which 178 studies were excluded, mainly because they did not report on any of the exposure features of interest. The remaining 25 studies were assessed for quality using the CASP tool after which 9 studies were excluded, resulting in a total of 16 studies included in the review.

\section{Description of the included studies}

The 16 studies included in this review are presented in Table 2. Eight studies were cohort studies of which 5 studies had obtained data during one single outbreak and 3 studies had collected data during two to six years. Six studies were of cross-sectional design and two studies were case-control studies. None of the included studies were of qualitative or mixed-methods design. The majority of studies $(n=11)$ covered COVID-19 outbreaks, two studies were on influenza outbreaks, two on gastrointestinal outbreaks, and one study on both respiratory and gastrointestinal outbreaks. Ten studies were undertaken in North America, one in Hong Kong and five in Europe. 
Table 2

Characteristics of studies included

\begin{tabular}{|c|c|c|c|c|c|c|c|}
\hline $\begin{array}{l}\text { Author, } \\
\text { year }\end{array}$ & $\begin{array}{l}\text { Country } \\
\text { (State/Region) }\end{array}$ & $\begin{array}{l}\text { Study design and } \\
\text { study period }\end{array}$ & Data source & Study setting & $\begin{array}{l}\text { Number } \\
\text { of care } \\
\text { homes }\end{array}$ & $\begin{array}{l}\text { Infectious } \\
\text { disease }\end{array}$ & Outcome of interest \\
\hline $\begin{array}{l}\text { Bowblis, J. } \\
\& \\
\text { Applebaum, } \\
\text { R. (2020) } \\
{[23]}\end{array}$ & $\begin{array}{l}\text { United States of } \\
\text { America (USA), } \\
\text { (Ohio) }\end{array}$ & $\begin{array}{l}\text { Cohort study, } \\
\text { data collected } \\
\text { April-June } 2020\end{array}$ & $\begin{array}{l}\text { Ohio Department of } \\
\text { Health, Nursing } \\
\text { Home Compare } \\
\text { database, and } \\
\text { Payroll-based } \\
\text { Journaling }\end{array}$ & $\begin{array}{l}\text { Nursing homes that } \\
\text { have reported at } \\
\text { least one case of } \\
\text { COVID- } 19 \text { to Ohio } \\
\text { Department of } \\
\text { Health. }\end{array}$ & 942 & COVID-19 & $\begin{array}{l}\text { Facility size, facility } \\
\text { location, temporary } \\
\text { hired staff, nurse } \\
\text { aides hours }\end{array}$ \\
\hline $\begin{array}{l}\text { Drinka P.J. } \\
\text { et al. } \\
(2004)[35]\end{array}$ & $\begin{array}{l}\text { USA } \\
\text { (Wisconsin) }\end{array}$ & $\begin{array}{l}\text { Cohort study, } \\
\text { data collected } \\
\text { during six } \\
\text { influenza season } \\
\text { 1993-2000 }\end{array}$ & $\begin{array}{l}\text { Objectilvey } \\
\text { measured data } \\
\text { collected by } \\
\text { researchers }\end{array}$ & $\begin{array}{l}\text { A four-building, } \\
\text { long-term care } \\
\text { facility for veterans } \\
\text { and their spouses }\end{array}$ & 1 & Influenza A & Facility design \\
\hline $\begin{array}{l}\text { Gorges, R.J. } \\
\text { \& Konetzka } \\
\text { R.T. }(2020) \\
\text { [37] }\end{array}$ & USA & $\begin{array}{l}\text { Cohort study, } \\
\text { data obtained in } \\
\text { June } 2020\end{array}$ & $\begin{array}{l}\text { Nursing Home } \\
\text { Compare (NHC) } \\
\text { database, Long-Term } \\
\text { Care Focus, and } \\
\text { COVID-19 Nursing } \\
\text { Home Dataset from } \\
\text { the Centers for } \\
\text { Medicare \& Medicaid } \\
\text { Services (CMS) }\end{array}$ & $\begin{array}{l}\text { All nursing homes } \\
\text { in the CMS COVID- } \\
19 \text { Nursing Home } \\
\text { Dataset that had } \\
\text { passed the CMS } \\
\text { Quality Assurance } \\
\text { Check as of June } \\
25,2020 \text {. }\end{array}$ & 13,167 & COVID-19 & Nurse aides hours \\
\hline $\begin{array}{l}\text { Halloran, } \\
\text { N.F. et al. } \\
(2020)[24]\end{array}$ & $\begin{array}{l}\text { England } \\
\text { (Cheshire and } \\
\text { Merseyside) }\end{array}$ & $\begin{array}{l}\text { Case-control } \\
\text { study comparing } \\
\text { characteristics } \\
\text { between care } \\
\text { homes with and } \\
\text { without a } \\
\text { declared } \\
\text { influenza } \\
\text { outbreak }\end{array}$ & $\begin{array}{l}\text { Public Health of } \\
\text { England Health } \\
\text { Protection Case } \\
\text { Management } \\
\text { System, and the Care } \\
\text { Quality Commission } \\
\text { (CQC) }\end{array}$ & $\begin{array}{l}\text { All CQC-registered } \\
\text { care homes in } \\
\text { Cheshire and } \\
\text { Merseyside that } \\
\text { declared an } \\
\text { influenza outbreak } \\
\text { between mid- } \\
\text { December } 2017 \\
\text { and May } 2018\end{array}$ & 154 & $\begin{array}{l}\text { Influenza A and } \\
\text { B }\end{array}$ & Facility size \\
\hline $\begin{array}{l}\mathrm{He}, \mathrm{M} \text { et al. } \\
(2020)[25]\end{array}$ & USA (California) & $\begin{array}{l}\text { Cross-sectional } \\
\text { study, data } \\
\text { obtained in June } \\
2020\end{array}$ & $\begin{array}{l}\text { California } \\
\text { Department of } \\
\text { Public Health, } \\
\text { Nursing Home } \\
\text { Compare Database, } \\
\text { and nursing home } \\
\text { data from Long- } \\
\text { Term Care Focus }\end{array}$ & $\begin{array}{l}\text { All nursing homes } \\
\text { in California }\end{array}$ & 1,223 & COVID-19 & $\begin{array}{l}\text { Facility size, facility } \\
\text { design }\end{array}$ \\
\hline $\begin{array}{l}\text { Inns, T. et } \\
\text { al. (2018) } \\
\text { [33] }\end{array}$ & $\begin{array}{l}\text { England } \\
\text { (Cheshire and } \\
\text { Merseyside) }\end{array}$ & $\begin{array}{l}\text { Cohort study, } \\
\text { data collected } \\
\text { December 2012- } \\
\text { December } 2016\end{array}$ & $\begin{array}{l}\text { Records collected by } \\
\text { the local Community } \\
\text { Infection Prevention } \\
\text { \& Control Team, and } \\
\text { data from the Care } \\
\text { Quality Commission } \\
\text { (CQC) }\end{array}$ & $\begin{array}{l}\text { All CQC-registered } \\
\text { care homes in } \\
\text { Cheshire and } \\
\text { Merseyside }\end{array}$ & 379 & $\begin{array}{l}\text { Infectious } \\
\text { gastroenteritis }\end{array}$ & Facility size \\
\hline $\begin{array}{l}\text { Li, J. et al. } \\
\text { (1996) [26] }\end{array}$ & $\begin{array}{l}\text { USA (New York } \\
\text { State) }\end{array}$ & $\begin{array}{l}\text { Case-control } \\
\text { study (case=care } \\
\text { home with } \\
\text { outbreak), all of } \\
\text { year } 1992\end{array}$ & $\begin{array}{l}\text { New York State } \\
\text { Department of } \\
\text { Health, and survey } \\
\text { data completed by } \\
\text { care home managers }\end{array}$ & $\begin{array}{l}\text { All licensed nursing } \\
\text { homes in New York } \\
\text { State caring } \\
\text { primarily for older } \\
\text { people }\end{array}$ & 171 & $\begin{array}{l}\text { Respiratory } \\
\text { and } \\
\text { gastrointestinal } \\
\text { infection }\end{array}$ & $\begin{array}{l}\text { Facility size, staff } \\
\text { compartmentalizing }\end{array}$ \\
\hline $\begin{array}{l}\text { Lin, } H_{\text {. et al. }} \\
(2011) \text { [27] }\end{array}$ & Hong Kong & $\begin{array}{l}\text { Cohort study } \\
\text { from January } \\
2005 \text { until } \\
\text { December } 2007\end{array}$ & $\begin{array}{l}\text { Annual Territorywide } \\
\text { Infection Control } \\
\text { Checklist Survey, and } \\
\text { the Public Health } \\
\text { Information System }\end{array}$ & $\begin{array}{l}\text { All elderly homes } \\
\text { operating in Hong } \\
\text { Kong }\end{array}$ & 748 & Norovirus & Facility design \\
\hline $\begin{array}{l}\text { Lombardo } \\
\text { F.L. et al. } \\
(2020) \text { [28] }\end{array}$ & Italy & $\begin{array}{l}\text { Cross-sectional } \\
\text { study, survey } \\
\text { completed } \\
\text { March-May } 2020\end{array}$ & $\begin{array}{l}\text { Survey data provided } \\
\text { by care home } \\
\text { directors }\end{array}$ & $\begin{array}{l}\text { All nursing homes } \\
\text { in Italy }\end{array}$ & 1,356 & COVID-19 & Facility size \\
\hline $\begin{array}{l}\text { Morciano, } \\
\text { M. et al al } \\
(2021) \text { [29] }\end{array}$ & England & $\begin{array}{l}\text { Cohort study, } \\
\text { data obtained in } \\
\text { August } 2020\end{array}$ & $\begin{array}{l}\text { Data records from } \\
\text { the Care Quality } \\
\text { Commission }\end{array}$ & $\begin{array}{l}\text { All nursing homes } \\
\text { in England with } \\
\text { death notification } \\
\text { data }\end{array}$ & 4,428 & COVID-19 & Facility size \\
\hline $\begin{array}{l}\text { Rolland, Y. } \\
\text { et al. } \\
(2020)[36]\end{array}$ & $\begin{array}{l}\text { France (Haute- } \\
\text { Garonne) }\end{array}$ & $\begin{array}{l}\text { Cross-sectional } \\
\text { study, survey } \\
\text { completed in } \\
\text { March-May } 2020\end{array}$ & $\begin{array}{l}\text { Survey data provided } \\
\text { by care home } \\
\text { directors }\end{array}$ & $\begin{array}{l}\text { All long-term care } \\
\text { facilities in Haute- } \\
\text { Garonne }\end{array}$ & 124 & COVID-19 & $\begin{array}{l}\text { Temporary hired } \\
\text { staff, staff } \\
\text { compartmentalizing }\end{array}$ \\
\hline $\begin{array}{l}\text { Shallcross, } \\
\text { L. et al } \\
(2021) \text { [30] }\end{array}$ & England & $\begin{array}{l}\text { Cross-sectional } \\
\text { study, telephone- } \\
\text { survey completed } \\
\text { in May-June } \\
2020\end{array}$ & $\begin{array}{l}\text { Survey data provided } \\
\text { by care home } \\
\text { directors, SARS-CoV- } \\
2 \text { RT-PCR test results }\end{array}$ & $\begin{array}{l}\text { All long-term care } \\
\text { facilities for } \\
\text { individuals aged } \\
\geq 65 \text { years or }\end{array}$ & 5,126 & COVID-19 & $\begin{array}{l}\text { Facility size, } \\
\text { temporary hired } \\
\text { staff }\end{array}$ \\
\hline
\end{tabular}




\begin{tabular}{|c|c|c|c|c|c|c|c|}
\hline & & & & $\begin{array}{l}\text { providing dementia } \\
\text { care }\end{array}$ & & & \\
\hline $\begin{array}{l}\text { Shi, S. et al. } \\
(2020) \text { [38] }\end{array}$ & $\begin{array}{l}\text { USA (Boston, } \\
\text { Massachusetts) }\end{array}$ & $\begin{array}{l}\text { Retrospective } \\
\text { cohort study, } \\
\text { data collected } \\
\text { March-May } 2020\end{array}$ & $\begin{array}{l}\text { Medical records, } \\
\text { SARS-CoV-2 test } \\
\text { results, federally } \\
\text { mandated clinical } \\
\text { assessment data of } \\
\text { residents }\end{array}$ & $\begin{array}{l}\text { An approximately } \\
500 \text {-bed academic } \\
\text { long-term care } \\
\text { facility in Boston }\end{array}$ & 1 & COVID-19 & Residency of staff \\
\hline $\begin{array}{l}\text { Stall, N. et } \\
\text { al. }(2020) \\
{[31]}\end{array}$ & $\begin{array}{l}\text { Canada } \\
\text { (Ontario) }\end{array}$ & $\begin{array}{l}\text { Retrospective } \\
\text { cohort study, } \\
\text { data collected } \\
\text { March-May } 2020\end{array}$ & $\begin{array}{l}\text { Ontario Ministries of } \\
\text { Health and Long- } \\
\text { Term Care, COVID-19 } \\
\text { Ontario Census }\end{array}$ & $\begin{array}{l}\text { All long-term care } \\
\text { homes in Ontario }\end{array}$ & 623 & COVID-19 & $\begin{array}{l}\text { Facility size, facility } \\
\text { design }\end{array}$ \\
\hline $\begin{array}{l}\text { Sugg, M. et } \\
\text { al. (2020) } \\
\text { [34] }\end{array}$ & USA & $\begin{array}{l}\text { Cross-sectional } \\
\text { study of a } \\
\text { national cohort, } \\
\text { data obtained in } \\
\text { June } 2020\end{array}$ & $\begin{array}{l}\text { Centers for Medicare } \\
\& \text { Medicaid Services, } \\
\text { American } \\
\text { Community Survey, } \\
\text { the } 2010 \text { Census, } \\
\text { SARS-CoV-2 test } \\
\text { results }\end{array}$ & $\begin{array}{l}\text { All nursing homes } \\
\text { operating since } \\
2015\end{array}$ & 13,709 & COVID-19 & Facility location \\
\hline $\begin{array}{l}\text { White, E.M. } \\
\text { et al. } \\
\text { (2020) [32] }\end{array}$ & USA & $\begin{array}{l}\text { Cross-sectional } \\
\text { study, data } \\
\text { obtained in May } \\
2020\end{array}$ & $\begin{array}{l}\text { Genesis Healthcare } \\
\text { database, Long-Term } \\
\text { Care Focus, Nursing } \\
\text { Home Compare } \\
\text { database }\end{array}$ & $\begin{array}{l}\text { Skilled nursing } \\
\text { facilities by the } \\
\text { provider Genesis } \\
\text { HealthCare and } \\
\text { other providers } \\
\text { operating in states } \\
\text { providing data on } \\
\text { COVID-19 test } \\
\text { results }\end{array}$ & 3,357 & COVID-19 & Facility size \\
\hline
\end{tabular}

\section{Facility size}

Eleven studies reported on facility size in relation to risk of infection outbreak of which several reported on multiple aspects of facility size as shown in Table 3. Two studies reported no association between facility size and risk of infectious disease outbreak [23, 24]. Most studies (8 of 11) reported an association between larger facility size and greater risk of an outbreak [25-32]. Four studies also reported on larger outbreaks in relation to facility size [23, 24, 30, 31] of which all but one showed a lower risk of a large outbreak in larger care homes [30]. In one study the size of the care home was further associated with the duration of the outbreak with larger care homes having longer lasting outbreaks [33]. 
Findings on facility size

\begin{tabular}{|c|c|c|c|}
\hline $\begin{array}{l}\text { No } \\
\text { association } \\
\text { between } \\
\text { facility size } \\
\text { and infectious } \\
\text { disease } \\
\text { outbreak }\end{array}$ & Facility size and risk of an outbreak & Facility size and larger outbreaks & $\begin{array}{l}\text { Facility size and } \\
\text { the extent of the } \\
\text { outbreak }\end{array}$ \\
\hline $\begin{array}{l}\text { Bowblis } \\
(2020) \\
(\mathrm{N}=292) \\
\text { Number of } \\
\text { beds } \\
\text { consistently } \\
\text { showed no } \\
\text { association } \\
\text { with the } \\
\text { likelihood of } \\
\text { having at least } \\
\text { one resident } \\
\text { infected in } \\
\text { COVID-19. }\end{array}$ & $\begin{array}{l}\text { He }(2020)(\mathrm{N}=1,223) \text { Larger homes with higher bed } \\
\text { occupancy were positively associated with having one } \\
\text { or more COVID-19 case (OR: } 1.009,95 \% \mathrm{Cl} 1.006-1.012) \\
\text { and COVID-19 mortality (OR 1.006, } 95 \% \mathrm{Cl} 1.003-1.009) \text {. } \\
\text { Adjusted for ownership and years of operation. }\end{array}$ & $\begin{array}{l}\text { Bowblis (2020) }(\mathrm{N}=292) \text { Facilities with a larger number } \\
\text { of beds were less likely to report a high number of cases } \\
\text { at two of three time points (marginal effects for April: } \\
\text { mean }-0.012 \text {, SE } 0.010 \text {; May: mean }-0.025 \text {, SE }-0.012 \\
\text { (p<0.05); June; mean }-0.032 \text {, SE } 0.007(\mathrm{p}<0.01)) \text {. } \\
\text { Adjusted for facility structural, occupancy and payer- } \\
\text { mix, resident and case-mix characteristics, and rurality. }\end{array}$ & $\begin{array}{l}\text { Inns (2018) } \\
\text { ( } \mathrm{N}=379) \text { The size } \\
\text { of the facility } \\
\text { was associated } \\
\text { with the duration } \\
\text { of the outbreak } \\
\text { with larger care } \\
\text { homes having } \\
\text { longer lasting } \\
\text { outbreaks (IRR } \\
1.426,95 \% \mathrm{Cl} \\
1.275-1.595 \text {, } \\
\text { p<0.001). } \\
\text { Adjusted for total } \\
\text { outbreaks, winter } \\
\text { outbreaks, care } \\
\text { home quality } \\
\text { rating, bed-to- } \\
\text { staff ratio, } \\
\text { residents with } \\
\text { dementia and } \\
\text { closure of home } \\
\text { within } 3 \text { days. }\end{array}$ \\
\hline
\end{tabular}

Halloran

(2020)

$(\mathrm{N}=154)$ No

difference in

the risks of an

influenza

outbreak

between

smaller homes

(<30 residents)

and larger

homes $(>30$

residents)

$(p=0.65)$.
Li (1996) ( $N=171)$ A greater number of beds was associated with nosocomial respiratory and gastrointestinal disease outbreaks (RR $1.005,95 \% \mathrm{Cl}$ 1.002-1.009). Adjusted for infection control actions such as medical protocols and laboratory results, and authorities' area offices.
Halloran (2020) ( $N=154)$ Compared to smaller facilities, larger facilities ( $\geq 51$ residents) had a lower risk of having residents with influenza like illness once an outbreak had been declared (RR $0.55,95 \% \mathrm{Cl} 0.38-0.80$, $\mathrm{p}<0.001$ ). Adjusted for dementia care, care home quality score and antiviral prophylaxis activation.
Lin (2011) (N=748) Higher rates of norovirus outbreaks observed in larger care homes (RR 1.4, 95\% Cl 1.3-1.5, $\mathrm{p}<0.0001)$. Adjusted for staff-to-resident ratio, age of residents, bedridden residents, wheelchair accessibility and partition between beds.
Shallcross (2021). $(N=5,126)$ No difference observed when comparing smaller care homes ( $<25$ beds) with larger care homes on the likelihood of a large outbreak (defined as $1 / 3$ residents infected) (25-50 beds OR 0.70 $95 \% \mathrm{Cl} 0.41-1.19 ;>50$ beds OR $1.13,95 \% \mathrm{Cl} 0.66-1.96$ ). Adjusted for social deprivation, provider type, staff-tobed ratio, region, quality rating, staff sick pay, cohorting of staff, cleaning frequency, use of personal protective equipment, inability to isolate residents, new admissions and closure to visitors.

Stall (2020) (N=623) Larger homes with more residents were protectively associated with the number of residents infected with COVID-19 (RR $0.84,95 \% \mathrm{Cl} 0.73$ 0.95 ) and resident deaths in COVID-19 (RR $0.81,95 \% \mathrm{Cl}$ $0.70-0.95)$. Adjusted for chain ownership and staff-tobed ratio.
Lomardo (2020) ( $\mathrm{N}=1,356)$ COVID-19 outbreak associated with facility size larger than the median of 60 beds (OR 1.50, 95\% Cl 1.09-2.07, p=0.013). Adjusted for lack of personal protective equipment, lack of personnel, lack of information, difficulty transferring, difficulty isolating, lack of medication, beds-to-staff ratio and geographical area.
Morciano $(2021)(\mathrm{N}=4,428)$ Larger homes had higher risk of COVID-19 deaths per bed: small homes $(0-23$ beds) OR 2.2, 95\% Cl 1.8-2.7; medium homes (24-40 beds) OR 4.7,95\% Cl 4.0-5.5; large homes (41+ beds) OR 8.6, 95\% Cl 7.3-10.0. Adjusted for dementia care, legal status and provider type.

Shallcross $(2021)(\mathrm{N}=5,126)$ Larger care homes were significantly more likely to have a COVID-19 outbreak compared to smaller care homes defined as $<25$ beds (reference). Care homes with $25-50$ beds OR 1.73 , $95 \% \mathrm{Cl} 1.30-2.31 ;>50$ beds OR $2.76,95 \% \mathrm{Cl} 1.97-3.88$ ). Adjusted for social deprivation, provider type, staff-tobed ratio, region, quality rating, staff sick pay, cohorting of staff, cleaning frequency, use of personal protective equipment, inability to isolate residents, new admissions and closure to visitors. 
odds of an outbreak (OR 1.38, 95\% Cl 1.18-1.61).

Adjusted for chain ownership and staff-to-bed ratio.

White $(2020)(\mathrm{N}=3,357)$ Larger facility (presented as a

10-bed difference in facility size) was associated with

greater probability of having at least one resident with

COVID-19 infection. Marginal effect: 0.90, SE 0.159,

$\mathrm{p}<0.001$. Findings adjusted for county COVID-19

prevalence, date of first county case, and universal

testing at facility.

Table 4 provides a summary of the findings on facility location, facility design, staff compartmentalizing, type of employment, nurse aides hours and residence of staff. 
Table 4

Findings on facility location, facility design, staff compartmentalizing, temporary hired staff, nurse aides hours, and residence of staff

\begin{tabular}{|c|c|c|c|c|c|c|c|}
\hline $\begin{array}{l}\text { Author, } \\
\text { year }\end{array}$ & $\begin{array}{l}\text { Any clarifications } \\
\text { related to the } \\
\text { outcome(s), and factors } \\
\text { adjusted for } \\
\text { statistically significant } \\
\text { associations }\end{array}$ & Facility location & $\begin{array}{l}\text { Facility } \\
\text { design }\end{array}$ & $\begin{array}{l}\text { Staff } \\
\text { compartmentalizing }\end{array}$ & $\begin{array}{l}\text { Temporary } \\
\text { hired staff }\end{array}$ & $\begin{array}{l}\text { Nurse } \\
\text { aides } \\
\text { hours per } \\
\text { resident } \\
\text { per day }\end{array}$ & $\begin{array}{l}\text { Residence } \\
\text { of staff }\end{array}$ \\
\hline $\begin{array}{l}\text { Bowblis, J. } \\
\text { \& } \\
\text { Applebaum, } \\
\text { R. (2020) }\end{array}$ & $\begin{array}{l}\text { Measures assessed at } \\
\text { three time points (April, } \\
\text { May, June 2020) which } \\
\text { were reported } \\
\text { separately and } \\
\text { combined. Findings } \\
\text { adjusted for facility } \\
\text { structural, occupancy } \\
\text { and payer-mix, resident } \\
\text { and case-mix } \\
\text { characteristics, and } \\
\text { rurality. }\end{array}$ & $\begin{array}{l}\text { Care homes in rural } \\
\text { areas, including rural } \\
\text { cities, were } \\
\text { consistently less likely } \\
\text { to have a COVID-19 } \\
\text { infected resident } \\
\text { (marginal effect mean } \\
\text { (SE) }-0.117 \text { ( } 0.057) \text {, } \\
\text { p<0.05). There was no } \\
\text { statistically significant } \\
\text { association at any of } \\
\text { the time points } \\
\text { between rural location } \\
\text { and having a high } \\
\text { number of COVID-19 } \\
\text { cases (defined as } \\
\text { number of cases } \\
\text { equal to at least } 20 \% \\
\text { of beds) among } \\
\text { facilities with at least } \\
\text { one case. }\end{array}$ & - & - & $\begin{array}{l}\text { No consistent } \\
\text { effects over } \\
\text { three months } \\
\text { were observed } \\
\text { for use of } \\
\text { temporary } \\
\text { hired staff on } \\
\text { the likelihood } \\
\text { of having a } \\
\text { resident with } \\
\text { COVID-19 } \\
\text { infection or } \\
\text { having a high } \\
\text { number of } \\
\text { COVID-19 } \\
\text { cases. }\end{array}$ & $\begin{array}{l}\text { No } \\
\text { consistent } \\
\text { effects } \\
\text { over three } \\
\text { months on } \\
\text { certified } \\
\text { nurse } \\
\text { aides } \\
\text { hours per } \\
\text { resident } \\
\text { day on the } \\
\text { likelihood } \\
\text { of having } \\
\text { a resident } \\
\text { with } \\
\text { COVID-19 } \\
\text { infection } \\
\text { or having } \\
\text { a high } \\
\text { number of } \\
\text { COVID-19 } \\
\text { cases. }\end{array}$ & - \\
\hline $\begin{array}{l}\text { Drinka P.J. } \\
\text { et al. } \\
(2004)\end{array}$ & $\begin{array}{l}\text { One building with more } \\
\text { space per resident and } \\
100 \% \text { filtered air } \\
\text { compared to three } \\
\text { (older) buildings with } \\
\text { fewer square feet per } \\
\text { resident and } 30-70 \% \text { air } \\
\text { circulated back into the } \\
\text { buildings. }\end{array}$ & - & $\begin{array}{l}\text { No } \\
\text { significant } \\
\text { differences } \\
\text { in infectious } \\
\text { outbreaks of } \\
\text { Influenza A } \\
\text { observed } \\
\text { between the } \\
\text { facility } \\
\text { buildings in } \\
\text { five } \\
\text { subsequent } \\
\text { years }\end{array}$ & - & - & - & - \\
\hline $\begin{array}{l}\text { Gorges, R.J. } \\
\text { \& Konetzka } \\
\text { R.T. (2020) }\end{array}$ & $\begin{array}{l}\text { High nursing aides } \\
\text { hours defined as } \\
\text { greater than 66th } \\
\text { percentile of case-mix } \\
\text { adjusted hours. } \\
\text { Adjusted for facility } \\
\text { size, ownership type, } \\
\text { chain status, } \\
\text { percentage of Medicaid } \\
\text { residents, percentage of } \\
\text { White residents, } \\
\text { metropolitan status, } \\
\text { and county cases per } \\
\text { capita. }\end{array}$ & - & - & - & - & $\begin{array}{l}\text { High nurse } \\
\text { aides } \\
\text { hours were } \\
\text { not } \\
\text { associated } \\
\text { with a } \\
\text { COVID-19 } \\
\text { outbreak } \\
\text { but } \\
\text { associated } \\
\text { with lower } \\
\text { risks for a } \\
\text { larger } \\
\text { outbreak } \\
\text { (OR 0.790, } \\
\text { SE 0.058, } \\
\text { p<0.01) }\end{array}$ & - \\
\hline $\begin{array}{l}\text { He, M et al. } \\
(2020)\end{array}$ & $\begin{array}{l}\text { Facility age measured } \\
\text { by years of operation. } \\
\text { Adjusted for facility } \\
\text { size and ownership } \\
\text { type. }\end{array}$ & - & $\begin{array}{l}\text { No } \\
\text { significant } \\
\text { associations } \\
\text { between } \\
\text { facility age } \\
\text { and one or } \\
\text { more cases } \\
\text { of COVID- } \\
19 .\end{array}$ & - & - & - & - \\
\hline $\begin{array}{l}\text { Li, J. et al. } \\
(1996)\end{array}$ & $\begin{array}{l}\text { Multiple units refer to } \\
\text { units in the same care } \\
\text { home. Findings } \\
\text { adjusted for infection } \\
\text { control actions such as } \\
\text { medical protocols and } \\
\text { laboratory results, and } \\
\text { authorities' area offices. }\end{array}$ & - & - & $\begin{array}{l}\text { Having staff working } \\
\text { at multiple units } \\
\text { increased the risk of a } \\
\text { nosocomial } \\
\text { respiratory or } \\
\text { gastrointestinal } \\
\text { disease outbreak (RR } \\
2.51,95 \% \mathrm{Cl} 1.07- \\
5.89 \text { ) compared to } \\
\text { having multiple units } \\
\text { with separate staff. }\end{array}$ & - & - & - \\
\hline $\begin{array}{l}\text { Lin, H. et al. } \\
(2011)\end{array}$ & $\begin{array}{l}\text { Number of outbreaks in } \\
\text { care homes that supply }\end{array}$ & - & $\begin{array}{l}\text { Having an } \\
\text { isolation }\end{array}$ & - & - & - & - \\
\hline
\end{tabular}


isolation areas for

infected residents part

of infection control

practices compared to

care homes with no

isolation areas area was

not

associated

with lower

risk of

norovirus

outbreaks.

\begin{tabular}{|c|c|}
\hline $\begin{array}{l}\text { Rolland, Y. } \\
\text { et al. } \\
(2020)\end{array}$ & $\begin{array}{l}\text { Staff } \\
\text { compartmentalization } \\
\text { defined as organization } \\
\text { of the work so that the } \\
\text { team works in small } \\
\text { groups in one area of } \\
\text { the care home with no } \\
\text { physical connection } \\
\text { with the other members } \\
\text { of the team. Type of } \\
\text { employment defined as } \\
\text { permanent versus use } \\
\text { of professional interim. } \\
\text { Findings adjusted for } \\
\text { care home } \\
\text { administrative status } \\
\text { and organization of the } \\
\text { meals. }\end{array}$ \\
\hline $\begin{array}{l}\text { Shallcross, } \\
\text { L. et al } \\
\text { (2021) }\end{array}$ & $\begin{array}{l}\text { Employment of other } \\
\text { bank or agency staff } \\
\text { used for nursing aides. } \\
\text { Findings adjusted for } \\
\text { social deprivation, } \\
\text { provider type, staff-to- } \\
\text { bed ratio, region, quality } \\
\text { rating, staff sick pay, } \\
\text { cohorting of staff, } \\
\text { cleaning frequency, use } \\
\text { of personal protective } \\
\text { equipment, inability to } \\
\text { isolate residents, new } \\
\text { admissions and closure } \\
\text { to visitors. }\end{array}$ \\
\hline
\end{tabular}

- S Staff

compartmentalization

was associated with

lower risk of COVID-

19 outbreak (OR 0.17

95\% Cl 0.04-0.67,

$\mathrm{p}<0.01$ ).

Use of

professional

interim was

not associated

with COVID-19

infection.

infection.

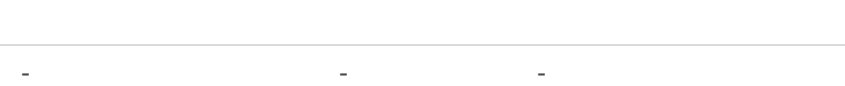

Temporary

employment a

few times per

month (OR

$1.28,95 \% \mathrm{Cl}$

1.20-1.37

$\mathrm{p}<0.0001), a$

few times per

week (OR 1.08,

$95 \% \mathrm{Cl} 1.01-$

$1.16, p=0.022)$

and on most

days or every

day (OR 1.08

$95 \% \mathrm{Cl} 1.00-$

$1.16, p=0.044)$

were all

associated

with higher

proportion

COVID-19

infection

among

residents

compared to

having no

temporary

staff.

Shi, S. et al. Home zip codes for all

(2020) staff were obtained to assess the proportion

of staff living in areas

with high rates of

COVID-19. Findings

adjusted for resident

characteristics (age,

sex, medical conditions,

activities of daily living

scores, bowel

incontinence, physical

behaviours, and

wandering.

$-$

Staff living

in a

community

with a high

rate of

COVID-19

was a

significant

predictor
of COVID-

19

infections

in the care

homes (OR

1.06

$95 \% \mathrm{Cl}$

1.04-1.08)

Stall, N. et Older design refers to

al. (2020) below year 1972 design

standards of larger

room size, private

washroom and single-

occupancy. Findings

adjusted for number of

residents, chain

ownership and staff-to-

bed ratio.

Older design -
standard (4-
person
rooms) was
associated
with greater
risk of
COVID-19
outbreaks
among
residents
(RR 1.88,
95\%Cl 1.27-
$2.79)$

Older design

standard (4-

among

residents

$95 \% \mathrm{Cl} 1.27$

Sugg, M. et Population density was A higher risk of

Page 9/14 
used as a proxy for urban location.

Findings adjusted for ownership, quality

rating, population employment rates, ethnic groups, household size, and income per capita.
COVID-19 outbreaks was observed in care homes located in areas with higher population density per square mile (rate ratio $1.10,95 \% \mathrm{Cl} 1.00-1.20$, $\mathrm{p}=0.042$ ) across the

USA. When examining

the results regionally,

the association

remained significant

in only 13 states.

\section{Facility location}

Two studies assessed the risk of a COVID-19 outbreak in relation to the care homes being in an urban or rural area [23, 34]. Both studies showed that care homes located in urban areas were more likely to report cases of COVID-19 of which one of the studies provided findings for 13,709 care homes across the USA [34]. In their study, they used Census 2010 data to calculate population density per square mile and used population density as a proxy for rurality. When examining the results regionally, the association remained significant in 13 states [34]. The other study was conducted in Ohio [23], one of the states in which the findings by Sugg et al. (2020) [34] remained when examined regionally. In the study from Ohio by Bowblis \& Applebaum (2020) [23], measures were taken once a month for three months (April-June 2019) and consistently showed that care homes in rural areas, including rural cities, were less likely to have a COVID-19 outbreak and less likely to have a larger outbreak (defined as at least $20 \%$ of residents infected) among facilities with at least one case.

\section{Facility design}

The influence on the facility design of the care homes on infectious disease outbreaks was examined in four studies focusing on various design aspects [25, $27,31,35]$. A study from the Canadian province Ontario showed that multi-occupancy rooms, more common in older than newer care homes, were associated with an almost two-fold risk of a COVID-19 outbreak compared to single rooms (RR 1.88,95\% Cl 1.27-2.79) [31]. In a study conducted in California, U.S., facility age measured as years of operation was not associated with an outbreak of one or more cases of COVID-19 (OR 1.006, 95\%CI 0.995-1.017) [25]. Similarly, another North American study also comparing older and newer buildings where the new building had installed filters preventing recirculation of air, showed no significant differences in influenza outbreaks observed between the buildings in five subsequent years [35]. Further, having isolation areas for infected residents to control infection was not associated with lower risk of norovirus outbreaks in a study of 748 care homes in Hong Kong [27].

\section{Staff compartmentalizing}

Two studies reported on staff compartmentalizing i.e. dividing staff at the care home facility into zones or units to prevent the spread of infection [26, 36]. One of the studies reported 2.5 times higher risks of nosocomial respiratory or gastrointestinal disease outbreaks if staff worked at multiple units compared to having staff compartmentalized (RR 2.51,95\% Cl 1.07-5.89) [26]. The other study was conducted during the COVID-19 pandemic and showed that arranging for staff compartmentalizing within zones resulted in a significantly lower likelihood of having any confirmed COVID-19 cases (OR 0.17, 95\%Cl 0.04-0.67) [36].

\section{Temporary hired staff}

Three studies reported on use of temporary hired staff and the likelihood of COVID-19 outbreaks in care homes [23, 30,36]. The largest of these three studies (care homes $n=5,126$ ) showed that use of temporary staff was associated with higher proportion COVID-19 infection among residents compared to having no temporary staff, with the strongest association in care homes that only had temporary staff a few times per month $(\mathrm{OR} 1.28,95 \% \mathrm{Cl} 1.20-1.37, \mathrm{p}<0.0001)$

[30]. The other two studies reported no association between use of temporary staff and COVID-19 outbreaks.

\section{Nurse aides hours}

The influence of nurse aides hours per resident on disease outbreaks was investigated in two studies of which both reported on COVID-19 outbreaks [23, 37]. Whilst one of the studies showed no consistent effects over three months of nurse aides hours and the number of COVID-19 cases [23], the other study reported no association between high nurse aides hours and an outbreak; yet showed an association between high nurse aides hours and lower risks for a larger COVID-19 outbreak [37].

\section{Residence of staff}

Only one study examined the influence of the residence of staff on infectious outbreaks in care homes. The study was conducted at a large care home (approximately 500 beds) and showed that staff living in a community with a high rate of COVID-19 was a significant predictor of COVID-19 outbreaks [38].

\section{Discussion}

In this review, a range of organisational facility and staff characteristics assessed in earlier studies on infectious disease outbreaks in care homes have been reported. The findings suggest that urban location and larger facility size may be associated with greater risks of an infectious outbreak. The findings also suggest that the risk of a larger outbreak may be lower in larger facilities. Whilst staff compartmentalizing may be associated with lower risk of outbreak, staff residing in highly infected areas may be associated with greater risk of an outbreak. The influence of facility design, use of temporary staff, and nurse aides hours on infectious outbreaks remains unclear. 
There is growing evidence that urban location and larger facilities increase the risk of infectious disease outbreaks in care homes [39, 40]. Larger care home facilities imply managing a greater number of residents including person-to-person contact with a larger number of different residents, staff and visitors, creating opportunities for infectious outbreaks [26]. The greater risk of infectious outbreaks that larger care homes seem to face may further reflect the community spread of infection [40]. As shown in this review, there is some evidence that staff residing in highly infected areas increases the risk of an outbreak at the care home where they work [38]. Previous research has also shown a link between the risk of an outbreak at a care home and the incidence of COVID-19 in the surrounding region [31]. Several earlier studies have implied that staff might contribute to the spread of infection [31, 40, 41]. Beside staff-toresident and staff-to-staff transmission at the same care home, many care workers often hold second jobs and provide care to family members, which may increase the risk of transmission [41]. They also tend to feel obligated to come to work even when ill, due to low incomes and limited benefits [41]. It has been speculated that spread of infection due to staff working at multiple facilities is most likely to occur among facilities using temporary staff who provide services at multiple care homes [23]. However, findings from the three included studies on use of temporary staff are inconsistent with only one study reporting such an association [30]. This might be explained by the unclarity in whether the outbreaks studied were caused by community transmissions or preexisting cases within the facility.

Apart from suggesting that larger facility size may be associated with greater risks of an infectious outbreak, this review also suggests that the risk of a larger outbreak seems lower in larger facilities. This might be explained by larger care homes often being purpose built allowing for staff compartmentalizing, suggesting that larger facilities have less crossover of staff between residents [24, 31]. Larger facilities might also have the advantage of 'economy of scale' in terms of greater resources (e.g. personnel and financial resources) allowing for high nurse aides hours. According to the findings of this systematic review, high nurse aides hours might not prevent outbreaks yet seem to reduce the risks of a larger outbreak. This supports a recent study showing that care homes that met the minimum staffing standards are likely to be able to prevent or delay COVID-19 resident infections [10]. Having enough nurse aides is considered crucial as implementing infection control such as laboratory testing and staff compartmentalizing at care homes is difficult without sufficient staffing levels [37]. This denotes strong and close links between facility and staff characteristics implying the need to apply a holistic perspective when studying disease outbreaks at care homes.

The included studies on facility design assessed different aspects: multi-occupancy rooms, older facility design, air circulation and isolation areas, aspects which all have been considered important in recent analyses of COVID-19 and the care home environment [9, 42]. Yet, the included studies reported no association on these aspects except multi-occupancy rooms, making it difficult to draw any conclusions on the influence of facility design on infectious outbreaks. Further well-designed studies are needed on the influence of the many aspects of facility design on infectious outbreaks to provide an understanding of their potential impact. Further research is also needed on staff and facility characteristics of large facilities in relation to infectious disease outbreaks, and should include tracking cases to provide possible explanations to this review's suggested findings on facility size.

\section{Strengths and limitations}

A major strength of the study is the novelty of systematically reviewing multiple organisational facility and staff characteristics on infectious disease outbreaks in care homes, a setting that has become increasingly important following the COVID-19 pandemic. The search strategy comprised of seven different outcome features of both facility and staff characteristics, was not restricted to a certain language or time period, and was applied to five different databases. Limitations include that the search did not include grey literature, possibly resulting in relevant data not being considered. Initially we intended to include care homes for older adults only however most studies did not specify the age of the residents making such restriction impossible. Further, whilst the search was not restricted to a certain infectious disease, most of the included studies focus on COVID-19, possibly negatively affecting the generalisability of the findings as COVID-19 has higher severe disease and mortality rates than e.g. influenza. However, not restricting the review to a certain infection is not necessarily a weakness per se. For instance, the lack of associations between facility design and infectious outbreaks in studies not focusing on COVID-19 might indicate that at least some aspects of facility design could be of greater importance to certain specific infections. Further, the methodological heterogeneity of the studies and the fact that the number of eligible studies was small makes it impossible to draw any firm conclusions. However, as the features addressed in the studies may have an impact and furthermore are amenable to interventions, further studies are strongly warranted.

\section{Conclusions, Implications And Further Research}

In conclusion, this systematic review suggests that larger facilities have greater risks of infectious outbreaks, yet the risk of a larger outbreak seems lower in larger facilities. This research could help informing the planning of future care homes and care home managers in their preparation of future infectious outbreaks. Following the COVID-19 pandemic, care home managers worldwide are preparing for new infectious outbreaks, however, evidence showing what actions on organisational characteristics are effective, is needed. Due to lack of robust findings the impact of facility and staff characteristics on infectious outbreaks remain largely unknown. Further research is needed to establish the effect of organisational characteristics of staff and facility on infectious disease outbreaks in care home

\section{Declarations}

\section{Acknowledgements}

We would like to thank librarians Carl Gornitzki and Jonas Pettersson for conducting the database searches.

\section{Ethics approval and consent to participate}

Not applicable. 


\section{Consent for publication}

Not applicable.

\section{Competing interests}

The authors declare that they have no competing interests and that the research was conducted in the absence of any commercial or financial relationships that could be construed as a potential conflict of interest.

\section{Funding}

The study is funded by The Swedish Research Council (Vetenskapsrådet) (2020-05850).

\section{Data availability}

All relevant data are included in the article and/or its supplementary information files.

\section{Authors' contributions}

AL and BB designed the study. Librarians Carl Gornitzki and Jonas Pettersson at Karolinska Institutet conducted the searches. AL and LM independently screened the abstracts. $A L$ and LM did the study selection, data extraction and quality assessment with input from JA. AL, LM, JA and BB planned the analyses and $A L$ and $L M$ undertook the analyses. All authors contributed to the interpretation of the findings. AL wrote the manuscript and all authors reviewed it. All authors have read and approved the manuscript.

\section{References}

1. Utsumi M, Makimoto K, Quroshi N, Ashida N. Types of infectious outbreaks and their impact in elderly care facilities: a review of the literature. Age and ageing. 2010;39(3):299-305.

2. Fallon A, Dukelow T, Kennelly SP, O'Neill D. COVID-19 in nursing homes. QJM : monthly journal of the Association of Physicians. 2020;113(6):391-2.

3. Lindsay L, Wolter J, De Coster I, Van Damme P, Verstraeten T. A decade of norovirus disease risk among older adults in upper-middle and high income countries: a systematic review. BMC infectious diseases. 2015;15:425.

4. Curran ET. Infection outbreaks in care homes: prevention and management. Nursing Times. 2017;113(9):18-21.

5. McMichael TM, Clark S, Pogosjans S, Kay M, Lewis J, Baer A, et al. COVID-19 in a Long-Term Care Facility - King County, Washington, February 27-March 9, 2020. MMWR Morbidity and mortality weekly report. 2020;69(12):339-42.

6. European Centre for Disease Prevention and Control. Surveillance of COVID-19 at long-term care facilities in the EU/EEA 2020. https://www.ecdc.europa.eu/en/publications-data/surveillance-COVID-19-long-term-care-facilities-EU-EEA. Accessed 9 Jun 2021.

7. Office for National Statistics. Deaths involving COVID-19 in the care sector, England and Wales: deaths registered between week ending 20 March 2020 and week ending 2 April 20212021.

https://www.ons.gov.uk/peoplepopulationandcommunity/birthsdeathsandmarriages/deaths/articles/deathsinvolvingcovid19inthecaresectorenglandand Accessed 10 Aug 2021.

8. Kaiser Family Foundation. State COVID-19 data and policy actions 2021. https://www.kff.org/coronavirus-covid-19/issue-brief/state-covid-19-data-andpolicy-actions/. Accessed 9 Jun 2021.

9. Wang Z. Use the Environment to Prevent and Control COVID-19 in Senior-Living Facilities: An Analysis of the Guidelines Used in China. HERD: Health Environments Research \& Design Journal. 2020:1937586720953519.

10. Harrington C, Ross L, Chapman S, Halifax E, Spurlock B, Bakerjian D. Nurse Staffing and Coronavirus Infections in California Nursing Homes. Policy, Politics, \& Nursing Practice. 2020;21(3):174-86.

11. de Boer B, Beerens HC, Katterbach MA, Viduka M, Willemse BM, Verbeek H. The Physical Environment of Nursing Homes for People with Dementia: Traditional Nursing Homes, Small-Scale Living Facilities, and Green Care Farms. Healthcare (Basel, Switzerland). 2018;6(4).

12. McClean P, Tunney M, Parsons C, Gilpin D, Baldwin N, Hughes C. Infection control and meticillin-resistant Staphylococcus aureus decolonization: the perspective of nursing home staff. Journal of Hospital Infection. 2012;81(4):264-9.

13. Mo S, Shi J. The Psychological Consequences of the COVID-19 on Residents and Staff in Nursing Homes. Work, aging and retirement. 2020;6(4):254-9.

14. Liddell K, Ruck Keene A, Holland A, Huppert J, Underwood BR, Clark O, et al. Isolating residents including wandering residents in care and group homes: Medical ethics and English law in the context of Covid-19. International journal of law and psychiatry. 2021;74:101649.

15. Xu D, Kane RL, Shamliyan TA. Effect of nursing home characteristics on residents' quality of life: a systematic review. Archives of gerontology and geriatrics. 2013;57(2):127-42.

16. Baldwin R, Chenoweth L, Dela Rama M, Wang AY. Does size matter in aged care facilities? A literature review of the relationship between the number of facility beds and quality. Health care management review. 2017;42(4):315-27.

17. Koshkouei M, Abel L, Pilbeam C. How can pandemic spreads be contained in care homes? : The Centre for Evidence-Based Medicine; 2020. https://www.cebm.net/covid-19/how-can-pandemic-spreads-be-contained-in-care-homes/. Accessed 9 Jun 2021. 
18. Mitchell BG, Gardner A, Stone PW, Hall L, Pogorzelska-Maziarz M. Hospital Staffing and Health Care-Associated Infections: A Systematic Review of the Literature. Joint Commission journal on quality and patient safety. 2018;44(10):613-22.

19. Ouslander JG, Grabowski DC. COVID-19 in Nursing Homes: Calming the Perfect Storm. J Am Geriatr Soc. 2020;68(10):2153-62.

20. Assariparambil AR, Nayak SG, Sugunan A, Mukhopadhyay C, Shankar R, Devi ES, et al. Structure, staffing, and factors affecting infection control nurses: Hospital based exploratory survey- A study protocol. Journal of advanced nursing. 2021;77(2):1062-9.

21. Chow EJ, Schwartz NG, Tobolowsky FA, Zacks RLT, Huntington-Frazier M, Reddy SC, et al. Symptom Screening at Illness Onset of Health Care Personnel With SARS-CoV-2 Infection in King County, Washington. Jama. 2020;323(20):2087-9.

22. Aschengrau A, Seage GR. Essentials of Epidemiology in Public Health. Fourth ed. Burlington: Jones and Bartlett Publishers, Inc.; 2020.

23. Bowblis J, Applebaum R. Prevalence of COVID-19 in Ohio Nursing Homes: What's Quality Got to Do with It? Journal of Aging \& Social Policy. $2020: 1-17$.

24. Halloran NF, Harries AD, Ghebrehewet S, Cleary P. Factors associated with influenza-like illness in care homes in Cheshire and Merseyside during the 20172018 influenza season. Public Health. 2020;187:89-96.

25. He M, Li Y, Fang F. Is There a Link between Nursing Home Reported Quality and COVID-19 Cases? Evidence from California Skilled Nursing Facilities. Journal of the American Medical Directors Association. 2020;21(7):905-8.

26. Li J, Birkhead GS, Strogatz DS, Coles FB. Impact of institution size, staffing patterns, and infection control practices on communicable disease outbreaks in New York State nursing homes. American Journal of Epidemiology. 1996;143(10):1042-9.

27. Lin H, Ng S, Chan S, Chan WM, Lee KC, Ho SC, et al. Institutional risk factors for norovirus outbreaks in Hong Kong elderly homes: a retrospective cohort study. BMC Public Health. 2011;11:297.

28. Lombardo FL, Bacigalupo I, Salvi E, Lacorte E, Piscopo P, Mayer F, et al. The Italian national survey on Coronavirus disease 2019 epidemic spread in nursing homes. International journal of geriatric psychiatry. 2020.

29. Morciano M, Stokes J, Kontopantelis E, Hall I, Turner AJ. Excess mortality for care home residents during the first 23 weeks of the COVID-19 pandemic in England: a national cohort study. BMC medicine. 2021;19(1):71.

30. Shallcross L, Burke D, Abbott O, Donaldson A, Hallatt G, Hayward A, et al. Factors associated with SARS-CoV-2 infection and outbreaks in long-term care facilities in England: a national cross-sectional survey. The Lancet Healthy longevity. 2021;2(3):e129-e42.

31. Stall NM, Jones A, Brown KA, Rochon PA, Costa AP. For-profit long-term care homes and the risk of COVID-19 outbreaks and resident deaths. CMAJ Canadian Medical Association Journal. 2020;192(33):E946-E55.

32. White EM, Kosar CM, Feifer RA, Blackman C, Gravenstein S, Ouslander J, et al. Variation in SARS-CoV-2 Prevalence in U.S. Skilled Nursing Facilities. Journal of the American Geriatrics Society. 2020;16:16.

33. Inns T, Keenan A, Huyton R, Harris J, Iturriza-Gomara M, O'Brien SJ, et al. How timely closure can reduce outbreak duration: gastroenteritis in care homes in North West England, 2012-2016. BMC Public Health. 2018;18(1):488.

34. Sugg MM, Spaulding TJ, Lane SJ, Runkle JD, Harden SR, Hege A, et al. Mapping community-level determinants of COVID-19 transmission in nursing homes: A multi-scale approach. Science of the Total Environment. 2020;752:141946.

35. Drinka PJ, Krause P. Report of an outbreak: nursing home architecture and influenza-A attack rates: update. Journal of the American Geriatrics Society. 2004;52(5):847-8.

36. Rolland Y, Lacoste MH, de Mauleon A, Ghisolfi A, De Souto Barreto P, Blain H, et al. Guidance for the Prevention of the COVID-19 Epidemic in Long-Term Care Facilities: A Short-Term Prospective Study. Journal of Nutrition, Health \& Aging. 2020;24(8):812-6.

37. Gorges RJ, Konetzka RT. Staffing Levels and COVID-19 Cases and Outbreaks in U.S. Nursing Homes. Journal of the American Geriatrics Society. 2020;08:08.

38. Shi SM, Bakaev I, Chen H, Travison TG, Berry SD. Risk Factors, Presentation, and Course of Coronavirus Disease 2019 in a Large, Academic Long-Term Care Facility. Journal of the American Medical Directors Association. 2020;21(10):1378-83.e1.

39. Abrams HR, Loomer L, Gandhi A, Grabowski DC. Characteristics of U.S. Nursing Homes with COVID-19 Cases. Journal of the American Geriatrics Society. 2020;68(8):1653-6.

40. Ochieng N, Chidambaram P, Garfield R, Neuman T. Factors Associated With COVID-19 Cases and Deaths in Long-Term Care Facilities: Findings from a Literature Review: Kaiser Family Foundation; 2021. https://www.kff.org/coronavirus-covid-19/issue-brief/factors-associated-with-covid-19-cases-anddeaths-in-long-term-care-facilities-findings-from-a-literature-review/. Accessed 9 Jun 2021.

41. Van Houtven CH, DePasquale N, Coe NB. Essential Long-Term Care Workers Commonly Hold Second Jobs and Double- or Triple-Duty Caregiving Roles. J Am Geriatr Soc. 2020;68(8):1657-60.

42. Anderson DC, Grey T, Kennelly S, O'Neill D. Nursing Home Design and COVID-19: Balancing Infection Control, Quality of Life, and Resilience. J Am Med Dir Assoc. 2020;21(11):1519-24.

\section{Figures}




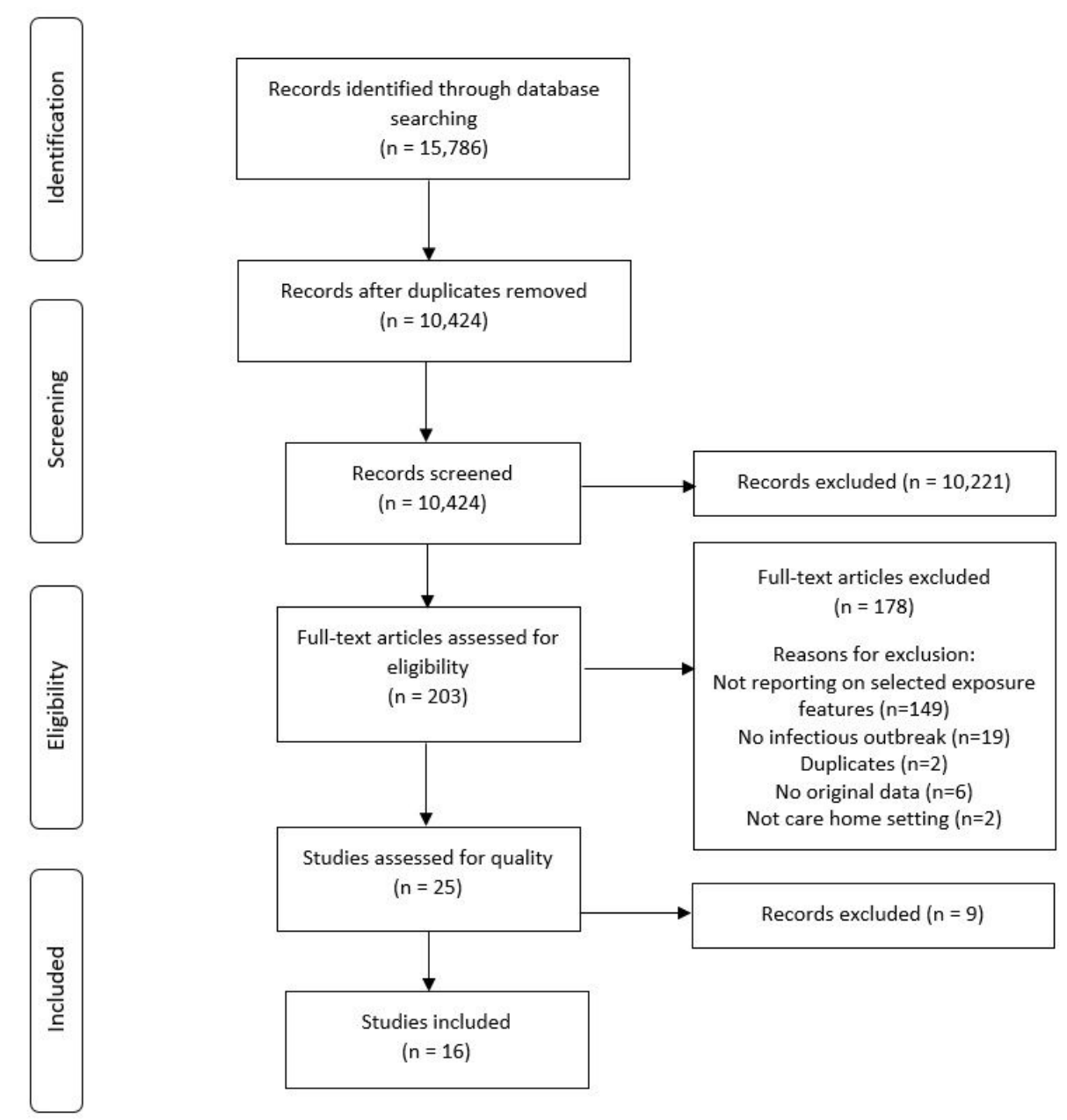

Figure 1

PRISMA flow chart

\section{Supplementary Files}

This is a list of supplementary files associated with this preprint. Click to download.

- Appendix1.Searchtermsused.docx 